\title{
Microscopic structure inputs for reaction models of fission
}

\author{
H. Goutte ${ }^{\text {a }, ~ A . ~ D o b r o w o l s k i, ~ M . ~ G i r o d, ~ a n d ~ J .-P . ~ D e l a r o c h e ~}$ \\ CEA/DAM Île-de-France, DPTA/Service de Physique Nucléaire, BP. 12, 91680 Bruyères-le-Châtel, France
}

\begin{abstract}
Reliable nuclear structure predictions are needed for reaction data evaluations, whenever experimental information is missing. Even if full parameter-free evaluations are not possible nowadays, some noteworthy progress in both structure and reaction fields has been accomplished recently. We present here some results on fission barriers and shape isomers partial $\gamma$-back decay and fission lifetimes. Calculations have been performed using the effective D1S Gogny force and the Generator Coordinate Method with the Gaussian Overlap Approximation based on HartreeFock-Bogoliubov results.
\end{abstract}

\section{Introduction}

As pointed out by M. Herman in ref. [1], nuclear reaction models should:

- provide data when experimental results are missing,

- help discriminate between experimental results,

- and ensure internal consistency of evaluations.

For all these reasons, a major goal nowadays is to remove step by step phenomenology in nuclear reaction models by using microscopic inputs built from nuclear structure information. We present here some recent results obtained for fission barriers [2] and shape isomers partial $\gamma$-back decay and fission lifetimes [3]. Calculations have been performed using the D1S Gogny force and the Generator Coordinate Method Approach with the Gaussian Overlap Approximation based on Constrained-Hartree-Fock-Bogoliubov states.

\section{Static least energy fission barriers}

In most of the self-consistent mean-field studies of fission barriers, nuclear shapes are generated by only the axial mass quadrupole moment $\hat{Q}_{20}$. The intrinsic deformed nuclear states are then solutions of the Constrained-Hartree-FockBogoliubov (CHFB) equation:

$$
\delta\left\langle\Phi\left(q_{20}\right)\left|\hat{H}-\lambda_{N} \hat{N}-\lambda_{Z} \hat{Z}-\lambda_{20} \hat{Q}_{20}\right| \Phi\left(q_{20}\right)\right\rangle=0,
$$

where the Lagrange parameters $\lambda_{N}, \lambda_{Z}$, and $\lambda_{20}$ are deduced from:

$$
\begin{gathered}
\left\langle\Phi\left(q_{20}\right)|\hat{N}| \Phi\left(q_{20}\right)\right\rangle=N, \\
\left\langle\Phi\left(q_{20}\right)|\hat{Z}| \Phi\left(q_{20}\right)\right\rangle=Z, \\
\left\langle\Phi\left(q_{20}\right)\left|\hat{Q}_{20}\right| \Phi\left(q_{20}\right)\right\rangle=q_{20} .
\end{gathered}
$$

In such an approach, all the deformations that are not constrained take on values that minimize total energy. In ref. [3] we have calculated such fission barriers for 55 even-even

\footnotetext{
${ }^{a}$ Presenting author, e-mail: heloise.goutte@cea.fr
}

actinides from ${ }^{226} \mathrm{Th}$ to ${ }^{262} \mathrm{No}$. Between the first and the second wells the triaxial degree of freedom has been left free so that triaxial inner barriers are obtained, whereas beyond the second well parity has been broken instead in the CHFB calculations. The main features of these fission barriers are:

- triaxial inner barriers are systematically lowered by up to $4 \mathrm{MeV}$ when compared to the axial ones,

- inner barrier heights display maxima at $\mathrm{N}=146$ for the uranium, plutonium, and curium isotopic chains,

- the outer barrier is found to be asymmetric for systems with $\mathrm{N}<152$ and symmetric for more neutron-rich systems,

- and finally super deformed minima appear to be washed out for $\mathrm{N}>156$.

Let us note that along such "least energy barriers" some high order multipole moments may display discontinuities when the system jump from one valley to another one. This feature is illustrated in figure 1, where CHFB energy is plotted as a function of axial quadrupole and hexadecapole deformation parameters, $\beta_{20}$ and $\beta_{40}$, respectively. The one-dimensional axial path obtained by constraining the axial quadrupole deformation and minimizing the energy is plotted as a dark line. The main feature is that a discontinuity is found for $\beta_{20} \simeq 0.50$, where $\beta_{40}$ drops from 0.35 to 0.15 .

Such discontinuities in high-order multipoles can affect single level properties. As an example, we have plotted in figure 2 the neutron single particle energies calculated along the axial deformation in ${ }^{238} \mathrm{U}$. The Fermi energy is marked as a thick grey curve. We clearly see that structure effects are observed around $\beta_{20} \simeq 0.5$, both in the single particle energies and in the Fermi energy. Such discontinuities may create artificial gaps for different elongations and should be carefully identified.

\section{Least action path}

In order to take into account mass parameters in the definition of the fission barriers and eventually to smooth discontinuities discussed above, least action paths (LAPs) have been determined. 


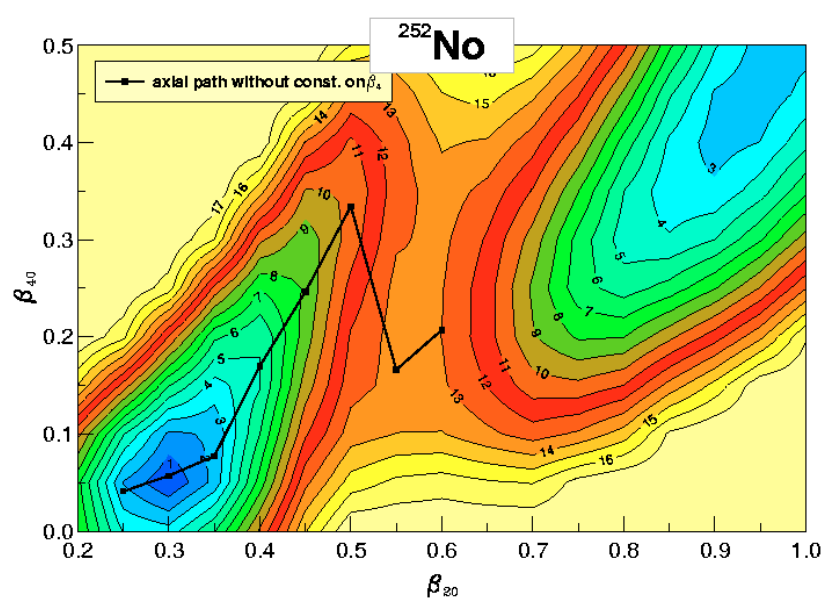

Fig. 1. CHFB energies as functions of axial quadrupole and hexadecapole deformation parameters, $\beta_{20}$ and $\beta_{40}$, respectively. Isolines are separated by $1 \mathrm{MeV}$. The one-dimensional axial path is plotted as a dark line.

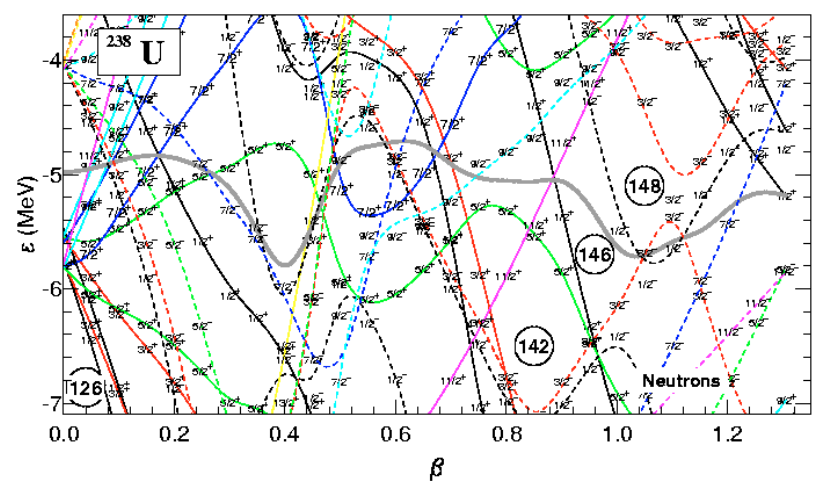

Fig. 2. Single particle energies $(\mathrm{MeV})$ for neutrons calculated along axial quadrupole deformation for ${ }^{238} \mathrm{U}$. Fermi energy is shown as a thick line. Dashed and solid lines are for $\pi=-$ and $\pi=+$ levels, respectively.

For that purpose, two dimensional landscapes are first determined from $\mathrm{CHFB}$ calculations:

$$
\delta\left\langle\Phi\left(q_{1}, q_{2}\right)\left|\hat{H}-\lambda_{N} \hat{N}-\lambda_{Z} \hat{Z}-\sum_{i=1,2} \lambda_{i} \hat{Q}_{i}\right| \Phi\left(q_{1}, q_{2}\right)\right\rangle=0,
$$

with

$$
\left\langle\Phi\left(q_{1}, q_{2}\right)|\hat{N}(\hat{Z})| \Phi\left(q_{1}, q_{2}\right)\right\rangle=N(Z)
$$

and

$$
\left\langle\Phi\left(q_{1}, q_{2}\right)\left|\hat{Q}_{i}\right| \Phi\left(q_{1}, q_{2}\right)\right\rangle=q_{i},
$$

where in the present calculations $\hat{Q}_{i(i=1,2)}$ are either i) $\hat{Q}_{20}$ and $\hat{Q}_{22}$ generating axial and triaxial quadrupole deformations, respectively, or ii) $\hat{Q}_{20}$ and $\hat{Q}_{30}$, where $\hat{Q}_{30}$ is for octupole deformation.

Least action paths in the axial $\left(\beta_{0}\right)$ and triaxial $\left(\beta_{2}\right)$ plane between the first well and the superdeformed one are displayed in figure 3 as black lines for uranium isotopes, and compared with least energy paths displayed as grey thick-lines. We clearly see that in the heavy uranium isotopes (A > 232), LAPs go through axial saddles, whereas they go through triaxial
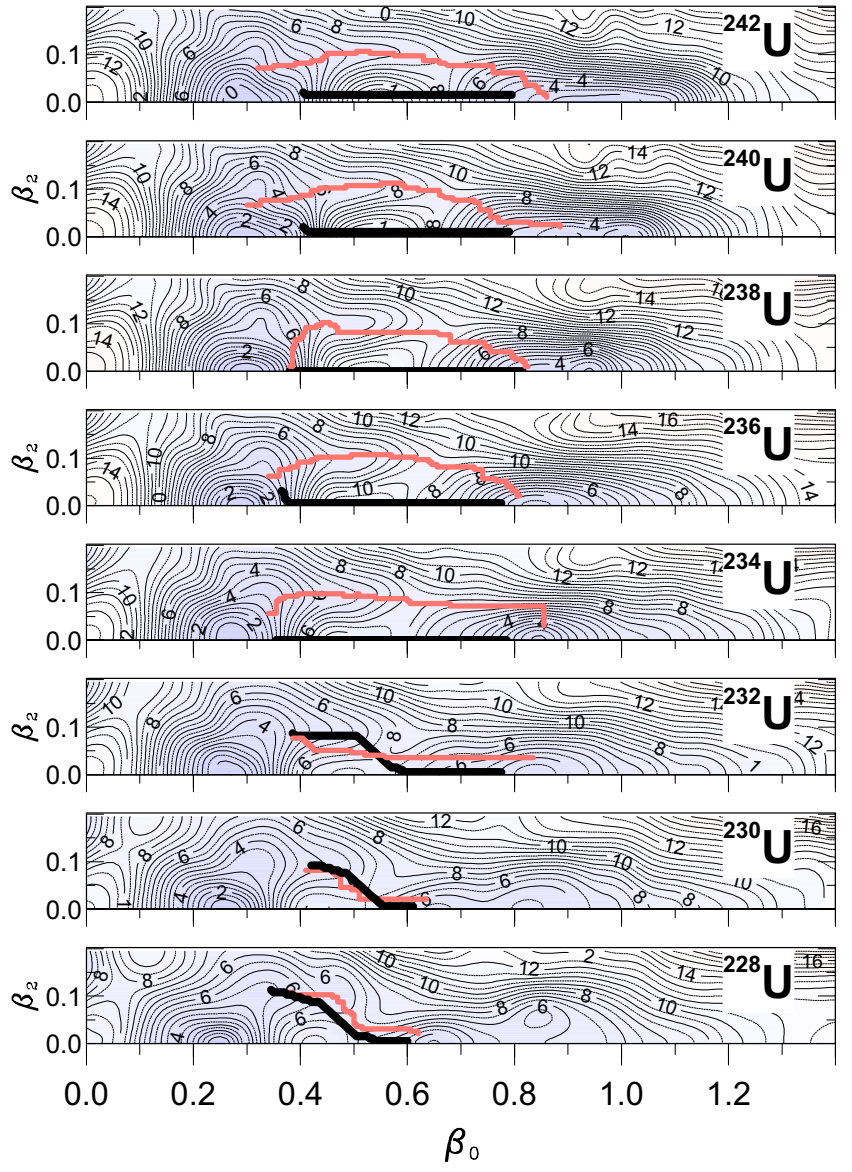

Fig. 3. CHFB energies as functions of the quadrupole axial and triaxial deformations, $\beta_{0}$ and $\beta_{2}$, respectively. Isolines are separated by $1 \mathrm{MeV}$. Least energy (least action) paths are shown as grey (black) thick-lines. See [3] for more details.

shapes in the lighter isotopes. Axial LAPs are also predicted in ${ }^{232-236} \mathrm{Th},{ }^{238-244} \mathrm{Pu},{ }^{240-246} \mathrm{Cm}$, and in all the $\mathrm{Cf}$, Fm and No isotopes exhibiting shape isomers. This illustrates how important is the role played by collective masses.

Least action paths from outer saddle point up to scission have been studied in the quadrupole, octupole plane. Results are presented in figures 4 and 5 for the ${ }^{226} \mathrm{Th}$ and ${ }^{238} \mathrm{U}$ isotopes, where least energy paths (solid lines) are compared with least action paths (dashed lines). We clearly see that:

- the height of the least energy and least action fission barriers can be different,

- this energy difference depends on the nucleus under study.

In the ${ }^{226} \mathrm{Th}$ nuclide, where symmetric and asymmetric valleys are almost degenerated in energy, the inertia is predicted to play a predominant role, as the least action path is predicted to follow the symmetric valley up to a large elongation $\left(q_{20} \simeq 150 \mathrm{~b}\right)$ as illustrated in figure 6 . This effect is not predicted for the ${ }^{238} \mathrm{U}$ isotope, where least energy and least action fission barriers are the same.

As discussed in [2] the least action approach to fission path is well-suited to study the problem of transitions between various energy valleys separated from each other by a 


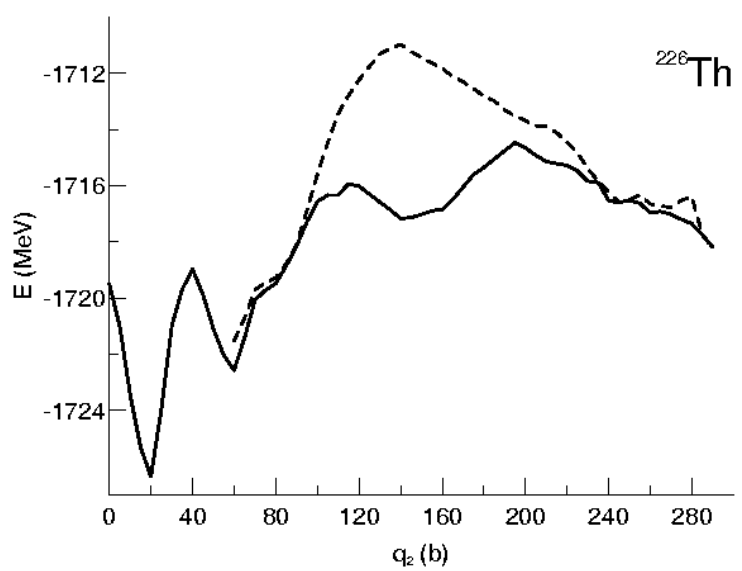

Fig. 4. CHFB energies as functions of the axial quadrupole moment $q_{20}$ (b) for the ${ }^{226} \mathrm{Th}$ isotope. Least energy path is displayed (solid line) and is compared with least action path (dashed line).

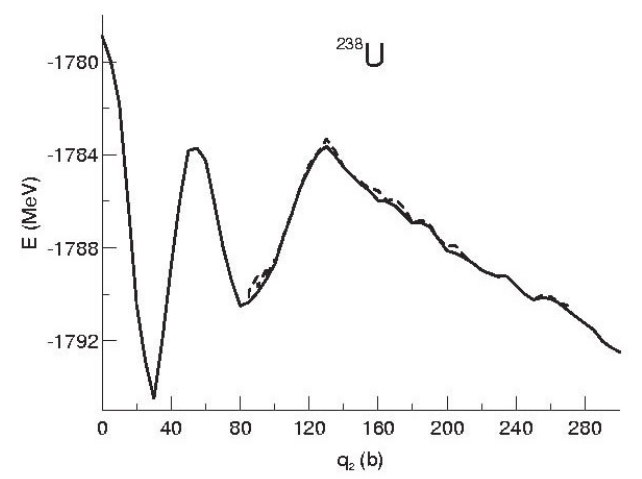

Fig. 5. Same as figure 4 for the ${ }^{238} \mathrm{U}$ isotope.

relatively low energy barrier and corresponding to different fission modes.

\subsection{Transverse vibrations}

In the LAP approach presented above, fluctuations around the path are not taken into account. In a more elaborated approach, we have considered the vibrational collective movements in the direction perpendicular to the fission path. In practice we have:

- defined a curvilinear absciss along the static path,

- defined the transverse directions all along the path,

- calculated inertia and potential energies in these transverse directions,

- calculated the transverse vibrations using the Generator Coordinate Method and the Gaussian Overlap Approximation,

- and finally defined the barrier as the lowest energy vibrational states for all curvilinear absciss.

Results are plotted in figures 7 and 8 for ${ }^{226} \mathrm{Th}$ and ${ }^{238} \mathrm{U}$, respectively. Least energy paths (solid lines) are compared with dynamical barriers (dashed lines), which take into account transverse vibrations. Let us mention that the curves

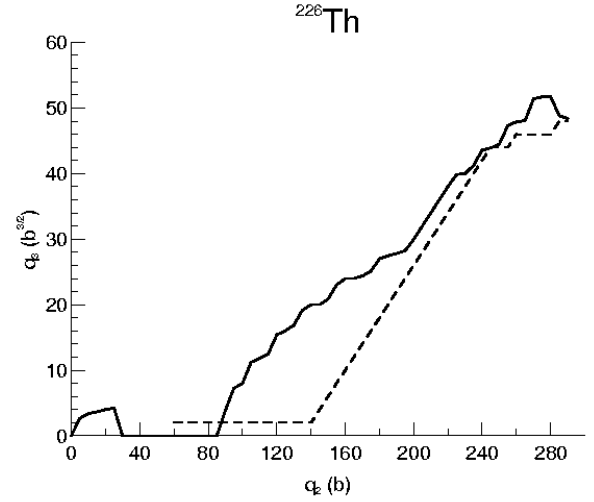

Fig. 6. Least energy (solid line) and least action (dashed line) paths for the ${ }^{226} \mathrm{Th}$ isotope over the quadrupole $\left(q_{20}\right)$ and octupole $\left(q_{30}\right)$ deformations.

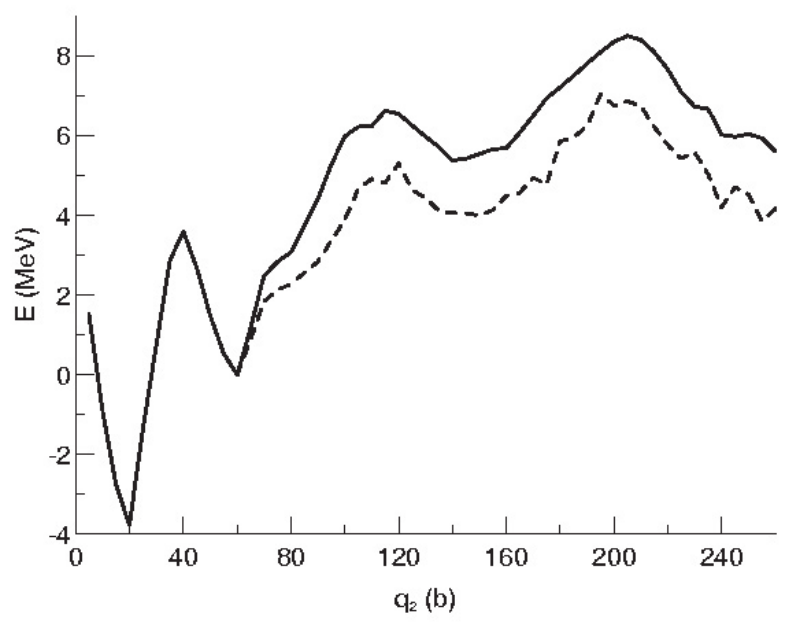

Fig. 7. CHFB energies as functions of the quadrupole moment for the ${ }^{226} \mathrm{Th}$ isotope. The least energy path (solid line) is compared with the dynamical barrier, which takes into account transverse vibrations (dashed line).

have been arbitrarly shifted in energy to coincide at the super deformed minimum. In the two studied nuclei, the dynamical barrier is found to be the lowest one, the energy difference being almost $1 \mathrm{MeV}$ for large elongations with $q_{20}>100 \mathrm{~b}$.

\section{Lifetime}

In order to analyze the importance of the inertia and of the transverse vibrations on barrier penetration, $\gamma$-back $\left(T_{\gamma}\right)$ and fission $\left(T_{f}\right)$ decay lifetime calculations have been performed. By use of the well-known one dimensional WKB formalism, partial half-lives $T_{\gamma,(f)}$ (in s) are calcualted as:

$$
T_{\gamma,(f)}=\frac{2 \cdot 87 \cdot 10^{-21}}{E_{0}}\left[1+e^{2 S_{\gamma,(f)}}\right]
$$

with $E_{0}$ (in $\mathrm{MeV}$ ) the assault energy and $S_{\gamma,(f)}$ the action calculated along the given trajectory $L_{\gamma,(f)}$ :

$$
S_{\gamma,(f)}=\int_{L_{\gamma,(f)}} \sqrt{2 M_{s}(s)\left[V(q(s))-E_{0}\right]} d s,
$$




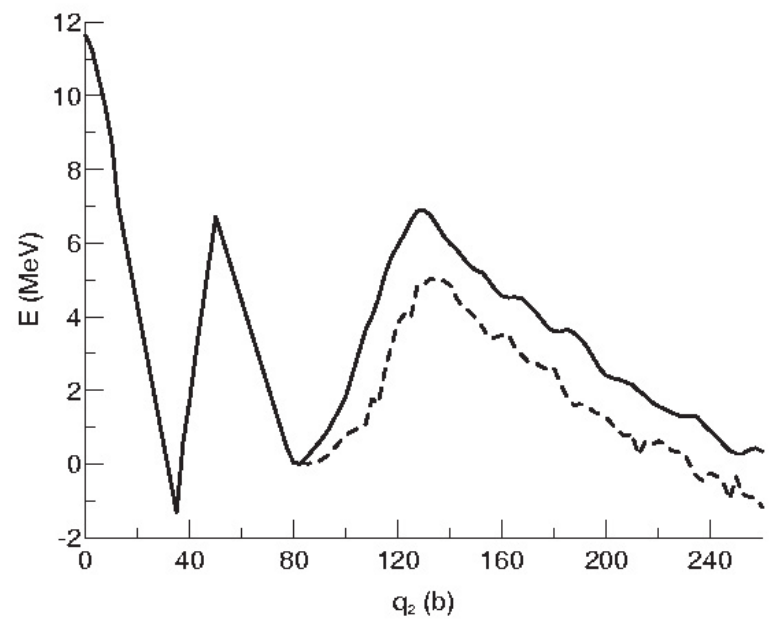

Fig. 8. Same as figure 7 for the ${ }^{238} \mathrm{U}$ isotope.

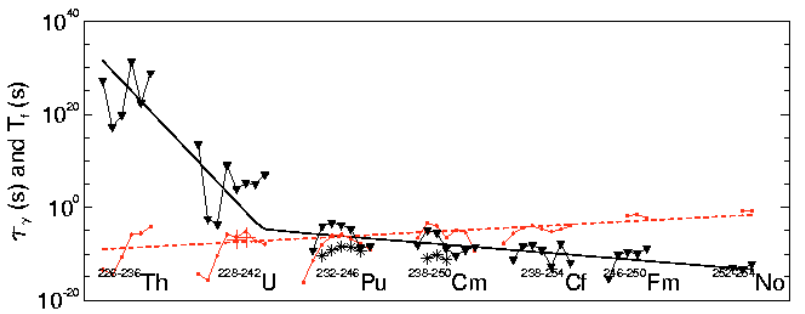

Fig. 9. Partial half-lives for $\gamma$-back and fission decays of shape isomers. Crosses $\left({ }^{236,238} \mathrm{U}\right)$ and stars $\left({ }^{236-244} \mathrm{Pu},{ }^{240-244} \mathrm{Cm}\right)$ are experimental data for $\gamma$-back and fission decays, respectively. Dots and triangles are one-dimension WKB predictions for $\gamma$-back and fission decays, respectively. Lines are plotted to guide the eye.

where $s$ is a curvilinear abscissa, and $M_{s}(s)$ is the effective inertia along $L_{\gamma,(f)}$ :

$$
M_{s}(s)=\sum_{i j} M_{i j}\left(q_{i}, q_{j}\right) \frac{d q_{i}}{d s} \frac{d q_{j}}{d s},
$$

where $M_{i j}$ are the collective vibrational mass parameters associated to the $q_{i}$ and $q_{j}$ degrees of freedom. Some calculations of shape isomer lifetimes have been performed and are shown in figure 9 where $\gamma$-back and fission decay results are displayed for 55 even-even actinides and are compared with experimental data when available. These lifetimes calculations have not been performed for the moment for the three different fission barriers discussed above, namely least-energy, leastaction and dynamical barriers including transverse vibrations. In the present calculations, the trajectories associated to the fission decay are taken as the lowest energy paths, whereas least action paths are considered in $\gamma$-back decay predictions.
Figure 9 shows that calculated fission lifetimes are found almost systematically longer than the experimental data, which are available only for $\mathrm{Pu}$ and $\mathrm{Cm}$ isotopes. On the contrary, calculated $\gamma$-back half-lives are found to be in qualitative agreement with measurements available for $U$ isotopes. The discrepancy for fission lifetime might be due to that least action paths have been ignored.

When comparing the $\gamma$-back and fission predicted lifetime values, we see that:

- shape isomers in Th and $\mathrm{U}$ isotopes predominantly decay through $\gamma$ emisison,

- fission and $\gamma$-back decays are competing for $\mathrm{Pu}$ and $\mathrm{Cm}$ elements,

- and finally, fission is by far dominating the decay processes for higher $\mathrm{Z}$ elements.

These overall predictions are in qualitative agreement with experimental data.

\section{Perspectives}

Nuclear fission is a complex process which involves the evolution of a nucleus over a multidimensional landscape. The problem of determining the most probable one-dimensional effective nuclear path, to be used in reaction model, is a subject of scientific effort since the discovery of the nuclear fission phenomenon. In recent works, we have determined fission barriers from mean-field-based calculations using the Gogny D1S force. Least energy barriers have been first determined and structure effects have been predicted in the actinide region, such as the disappearence of superdeformed minima for isotopes with $\mathrm{N}>156$. Then, least action paths have been calculated and the effect of the inertia has been discussed. Finally, dynamical barriers have been built using the transverse vibrational states obtained from the Generator Coordinate Method and the Gaussian Overlap Approximation. The first calculations of shape isomer half-lives in eveneven actinides have shown that $\gamma$-back and fission decays are competing in this region. In order to study in details these structure effects, and to analyze the influence of the inertia and of the transverse vibrations, lifetimes calculations should be performed for all the actinides with the three prescriptions of fission barriers discussed above. This will be done in a near future.

\section{References}

1. M. Herman, Radiochim. Acta 89, 305 (2001).

2. A. Dobrowolski, H. Goutte, J.-F. Berger, Int. J. Mod. Phys. E 16, 431 (2007).

3. J.-P. Delaroche, M. Girod, H. Goutte, J. Libert, Nucl. Phys. A 771, 103 (2006). 